\title{
Residual symbol timing offset estimation for DRM+ system with cyclic delay diversity
}

\author{
Eu-Suk Shim ${ }^{1}$, Hyun Yang ${ }^{1}$, Ki-Won Kwon ${ }^{2}$, \\ and Young-Hwan You ${ }^{1 a)}$ \\ ${ }^{1}$ uT Communication Research Institute, Sejong University, Korea \\ ${ }^{2}$ Advanced Mobile Technology Research Center, Korea Electronics Technology \\ Institute, Korea \\ a)yhyou@ sejong.ac.kr
}

\begin{abstract}
In this study, we propose an enhanced residual symbol timing offset (STO) estimation scheme in an orthogonal frequency division multiplexing (OFDM) based digital radio mondiale plus (DRM+) system with cyclic delay diversity (CDD). By proper selection of the amount of cyclic delay and pilot subset partitioning, a robust and lowcost residual STO estimator is derived. By computer simulation, the proposed estimator is shown to benefit from properly designed delay parameter and pilot subsets.
\end{abstract}

Keywords: DRM+, cyclic delay diversity, symbol timing offset, OFDM

Classification: Science and engineering for electronics

\section{References}

[1] ETSI ES 201980 V3.1.1, "Digital radio mondiale (DRM) - system specification," draft, Aug. 2009.

[2] A. Steil, F. Schad, M. Feilen, M. Kohler, J. Lehnert, E. Hedrich, and G. Kilian, "Digitising VHF FM sound broadcasting with DRM+ (DRM mode E)," Proc. Int. Symp. Broadband Multimedia Syst. Broadcast. 2009, pp. 1-6, 2009.

[3] A. Lodhi, F. Said, M. Dohler, and A. Aghvami, "Performance comparison of space-time block coded and cyclic delay diversity MC-CDMA systems," IEEE Wireless Commun., vol. 12, no. 2, pp. 38-45, April 2005.

[4] G. Bauch and J. S. Malik, "Cyclic delay diversity with bit-interleaved coded modulation in orthogonal frequency division multiple access," IEEE Trans. Wireless Commun., vol. 5, no. 8, pp. 2092-2100, Aug. 2006.

[5] S. Lu and N. Al-Dhahir, "A novel CDD-OFDM scheme with pilot-aided channel estimation," IEEE Trans. Wireless Commun., vol. 8, no. 3, pp. 1122-1127, March 2009.

[6] M. Speth, S. Fechtel, G. Fock, and H. Meyr, "Optimum receiver design for OFDM-based broadband transmission-Part II: A case study," IEEE Trans. Commun., vol. 49, no. 4, pp. 571-578, April 2001.

[7] B. Yang, K. B. Letaief, R. S. Cheng, and Z. Cao, "Timing recovery for OFDM transmission," IEEE J. Sel. Areas Commun., vol. 18, no. 11, 
pp. 2278-2291, Nov. 2000.

[8] A. I. Bo, G. E. Jian-hua, and W. Yong, "Symbol synchronization technique in COFDM systems," IEEE Trans. Broadcast., vol. 50, no. 1, pp. 56-62, March 2004.

[9] D.-C. Chang, "Effect and compensation of symbol timing offset in OFDM systems with channel interpolation," IEEE Trans. Broadcast., vol. 54, no. 4, pp. 761-770, Dec. 2008.

[10] Petition for Rulemaking to the United States Federal Communications Commission for In-Band On-Channel Digital Audio Broadcasting, USADR, Oct. 1998.

\section{Introduction}

Orthogonal frequency division multiplexing (OFDM) has been chosen as the transmission method for several broadcasting standards, including the digital audio broadcasting (DAB), digital radio mondiale (DRM), and terrestrial digital video broadcasting (DVB-T). While DRM currently covers the broadcasting bands below $30 \mathrm{MHz}$, its consortium is extending DRM to broadcasting bands up to $120 \mathrm{MHz}[1,2]$ by including the DRM robustness mode E, referred to as 'DRM+' in this paper.

In the DRM+ system, the bandwidth (BW) of approximately $100 \mathrm{KHz}$ is required to provide compatibility with FM broadcasting service and is quite small compared to the coherence BW of the channels which is in the order of $1 \mathrm{MHz}$ e.g. for typical urban environments [2]. Thus, the major challenge in the DRM+ is to cope with severe flat fading channels. One of possible solutions to combat flat fading is a cyclic delay diversity (CDD) approach, which is developed in $[3,4]$. Since the CDD scheme randomizes a channel frequency response, the frequency selectivity of the resulting channel transfer function increases, thereby significantly reducing the likelihood of deep fading. However, the increased frequency selectivity can be a problem for an interpolation-based channel estimation in the typical broadcasting system because it becomes more difficult to obtain an accurate interpolation between the pilot subcarriers in the presence of a residual symbol timing offset (STO) [5]. Hence, increasing the accuracy of residual STO estimation to obtain de-rotated fast Fourier transform (FFT) output signals for channel interpolation is one of the major concerns for the OFDM system with CDD.

In this paper, we propose an efficient estimation of residual STO in the OFDM-based DRM+ system with two CDD antennas. Cyclic delay selection and pilot partitioning are performed by exploiting a periodic nature of channel transfer function, and using these parameters will improve the performance of the residual STO estimation scheme and its implementation complexity is much lower than the conventional scheme.

This paper is organized as follows: Section 2 describes the signal model for the OFDM-based DRM+ system with using CDD. In Section 3, we briefly introduce a conventional residual STO estimation scheme and propose an efficient residual STO estimation scheme for the DRM+ system. In Section 4, 
we present simulation results illustrating the performance of the proposed estimator, and we conclude this paper with Section 5 .

\section{System description}

We consider an OFDM system with $N$ subcarrier and $N_{g}$ guard interval (GI) samples. When the system utilizes the CDD technique, the transmitted OFDM signal at the $t$-th transmit antenna can be expressed as

$$
x_{t}(n)=\frac{1}{\sqrt{N_{T}}} x\left(n-\delta_{t}\right)_{N}, t=1,2, \cdots, N_{T},
$$

where $N_{T}$ is the number of transmit antennas, $\delta_{t}$ is the amount of cyclic delay in the $t$-th antenna, and $(\cdot)_{N}$ is the modulo- $N$ operation. Assuming that the length $N_{g}$ of the GI represents the expected maximum length of the channel response, the cyclic delay could be chosen according to $\delta_{t+1}=N_{g}+1+\delta_{t}$, $t=1,2, \cdots, N_{T}$. As discussed in many literature [3, 4], another safe choice is $\delta_{t+1}=N / N_{T}+\delta_{t}, \quad t=1,2, \cdots, N_{T}$, which yields largest delay differences and zero correlation between adjacent $N_{T}$ subcarriers.

This paper focuses on the estimation of residual STO $\tau$ assuming that carrier frequency offset (CFO) has been eliminated. At the receiver end, the cyclic extension is removed and the received symbol is demodulated using the FFT operation. If $\tau$ is in the inter-symbol interference free region of the GI duration, the FFT output $Y(k)$ corresponding to the $k$-th subcarrier takes the expression $Y(k)=H(k) X(k) e^{-j 2 \pi k \tau / N}+W(k), \quad-N / 2 \leq$ $k \leq N / 2$, where $H(k)$ denotes the overall channel transfer function $H(k)=$ $1 / \sqrt{N_{T}} \sum_{t=1}^{N_{T}} H_{t}(k) e^{-j 2 \pi k \delta_{t} / N}, \tau$ is the residual STO normalized to sample interval, $H_{t}(k)$ is the channel frequency response from the $t$-th transmit antenna, and $W(k)$ is a complex Gaussian noise term.

The OFDM symbol in a frequency domain is assumed to be comprised of $N_{t r c}$ time reference cells (TRCs) and $N-N_{t r c}$ data subcarriers. For a simple description, carrier indices for TRCs is denoted by $\mathcal{S}=\left\{k_{1}, k_{2}, \cdots, k_{N_{t r c}}\right\}$, where $N_{t r c}$ is the number of TRCs and $k_{i}$ stands for the $i$-th pilot subcarrier.

\section{Residual STO estimation for DRM+ using CDD}

In this section, we briefly introduce a conventional residual STO estimation scheme when the TRC is used for pilot information and propose a way into estimating the residual STO with low complexity and improved performance for the DRM+ system using the CDD technique.

\subsection{Conventional residual STO estimation scheme}

The residual SFO is typically done by estimating the channel impulse response (CIR) time delay by the IFFT operation with zero padding at pilot subcarriers [6, 7], while another approach in $[8,9]$ calculates the rotated phase on scattered pilot subcarriers at the FFT output. The latter is usually used for in wireless broadcasting systems like DVB and DRM where the estimated channel responses of data subcarriers are obtained by interpolating 
the channel responses of scattered pilot subcarriers. As reported in [9], the pilot-based residual STO estimation method is computationally efficient but has larger estimation error than the estimation schemes $[6,7]$ which require intensive computations.

The basic concept in $[8,9]$ is effortlessly applied to the DRM+ system having the TRC set denoted by $\mathcal{S}=\{-80,-79,-77,-53,-52,-51,-32,-31$, $12,13,14,21,22,23,40,41,42,67,68,79,80\}$ [1]. By considering the pilots with index difference $\Delta_{k}=1$ in $\mathcal{S}$, the residual STO value can be estimated by

$$
\hat{\tau}=\frac{1}{2 \pi \Delta_{k} / N} \measuredangle\left\{\sum_{k \in \mathcal{S}_{\Delta_{k}}=1} \hat{Y}\left(k_{i}\right) \hat{Y}^{*}\left(k_{j}\right)\right\}
$$

where $\mathcal{S}_{\Delta_{k}=1}=\left\{\left(k_{i}, k_{j}\right) \mid \Delta_{k}=k_{i}-k_{j}=1\right\}$ stands for the set containing the elements of two pilots with index difference $\Delta_{k}=1, \measuredangle$ denotes the angle operation, and $\hat{Y}(k)=Y(k) X^{*}(k)$. Note that (2) is based on the assumption that choosing $\Delta_{k}$ as small as possible ensures $H(k) \approx H\left(k+\Delta_{k}\right)$, thereby $H(k) H^{*}\left(k+\Delta_{k}\right) \approx|H(k)|^{2}$. However, $H(k) \approx H\left(k+\Delta_{k}\right)$ is not guaranteed because of the increased frequency selectivity when the CDD is adopted in the DRM+ system.

\subsection{Proposed residual STO estimation scheme}

When the DRM+ system uses the CDD scheme to combat the flat fading effect, $\{H(k), k \in \mathcal{S}\}$ become highly uncorrelated even between adjacent subcarriers because of the effect of CDD. Thus, the conventional algorithm (2) can not cope with the dynamic change of the channel frequency response, giving rise to the performance degradation of SFO estimation. To solve this problem, an efficient residual STO estimation scheme by using the periodic nature of the equivalent single-input single-output channel $H(k)$ is suggested. Throughout this paper, we assume that $N_{T}=2$ and there is no cyclic delay at 1 st transmit antenna, i.e. $\delta_{1}=0$, without the loss of generality.

The basic concept of the proposed STO estimation scheme is based on that the $N_{t r c}$ TRCs are grouped into a number of subsets of pairs of two pilot subcarriers, so that every two pilots in each subset experience flat fading. Since the DRM+ system for FM broadcasting applications suffers from severe flat fading conditions [2], i.e. the channels $\left\{H_{t}(k)\right\}$ are assumed to be frequency-flat, one can effortlessly find that $H(k)$ is periodic with a period of $P_{H}=N / \delta_{2}$ when $N_{T}=2$ and $\delta_{1}=0$.

For algorithm description, let $\mathcal{S}_{\Delta_{m}, \delta}$ be the $m$-th subset containing the elements of two pilots with index difference $\Delta_{m}$ for a given integer-valued $\delta$, that is, $\mathcal{S}_{\Delta_{m}, \delta}=\left\{\left(k_{i}, k_{j}\right) \mid \Delta_{m}=k_{i}-k_{j}\right\}$. Denote $\mathcal{S}_{\delta}$ and $N_{s, \delta}$ to be the set of possible candidates of $\delta_{t}$ and the number of the subsets, respectively. The overall procedure for partitioning the TRC and choosing cyclic delay when $N_{T}=2$ is summarized as follows: 
Table I. Selected TRC subset and its number of elements.

\begin{tabular}{|c|c|c|c|c|c|c|c|c|c|}
\hline$m$ & 1 & 2 & 3 & 4 & 5 & 6 & 7 & 8 & 9 \\
\hline \hline$\Delta_{m}$ & 3 & 9 & 12 & 18 & 21 & 24 & 27 & 45 & 63 \\
\hline \# of pairs $\left(k_{i}, k_{j}\right)$ & 1 & 3 & 2 & 2 & 2 & 1 & 4 & 2 & 1 \\
\hline
\end{tabular}

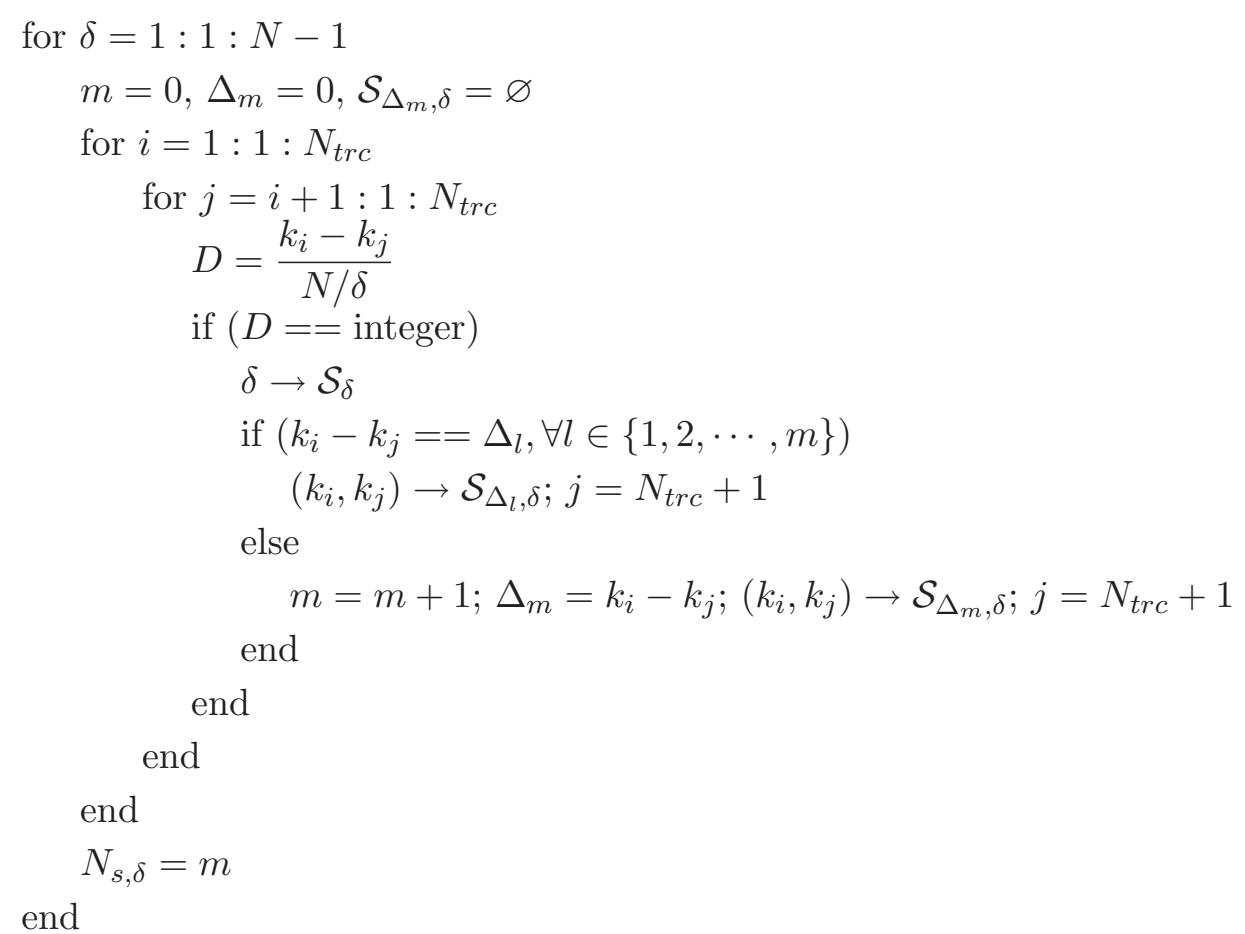

Note that integer-valued $D$ means that $k_{i}-k_{j}$ is an integer multiple of the periodicity of the channel $H(k)$. Therefore, any pair $\left(k_{i}, k_{j}\right)$ in $\mathcal{S}_{\Delta_{m}, \delta}$ experiences flat fading, i.e. $H\left(k_{i}\right) \approx H\left(k_{j}\right)$.

By a simple search taking account into the OFDM parameter and pilot pattern defined in the DRM+ system [1], one can effortlessly find that $N_{t r c}=$ 21 pilots are divided into 9 subsets having distinctive $\Delta_{m}$ 's and $\mathcal{S}_{\delta}=\{71\}$, thereby $N_{s, \delta}=9, \delta_{2}=71$, and $k_{i}-k_{j} \in\left\{\Delta_{1}, \Delta_{2}, \cdots, \Delta_{N_{s, \delta}}\right\}$. Table I shows the searched pilot subset and its number of elements.

For a robust and low-complexity STO estimation, we use the pilot subsets designed as in Table I. Without the loss of generality, we assume that $\Delta_{1}<$ $\Delta_{2}<\cdots<\Delta_{N_{s, \delta}}$. Based on the divided subsets, the estimator calculates the phase difference for each subset individually as follows

$$
\hat{\tau}=\frac{1}{N_{\max }} \sum_{m=1}^{N_{\max }} \frac{1}{2 \pi \Delta_{m} / N} \measuredangle\left\{\sum_{k \in \mathcal{S}_{\Delta_{m}, \delta}} \hat{Y}\left(k_{i}\right) \hat{Y}^{*}\left(k_{j}\right)\right\}
$$

where $N_{\max } \leq N_{s, \delta}$ stands for the number of pilot subset used to estimate STO. Note the the estimation range of (3) is limited by $|\tau| \leq N /\left(2 \Delta_{N_{\max }}\right)$, thereby is decreased with increase in $\Delta_{N_{\max }}$. Therefore, $N_{\max }$ is a fundamental design parameter that deals with trades-offs between the estimation range and performance. 
Table II. Complexity comparison of (2) and (3).

\begin{tabular}{|c|c|c|c|c|c|c|c|}
\hline \multirow{2}{*}{ Algorithm } & $(2)$ & \multicolumn{6}{|c|}{$N_{\max }$ in $(3)$} \\
\cline { 3 - 8 } & & 2 & 3 & 4 & 5 & 6 & 7 \\
\hline \hline Complex multiplication & 36 & 9 & 15 & 21 & 27 & 30 & 42 \\
\hline Real addition & 22 & 5 & 8 & 11 & 14 & 15 & 22 \\
\hline
\end{tabular}

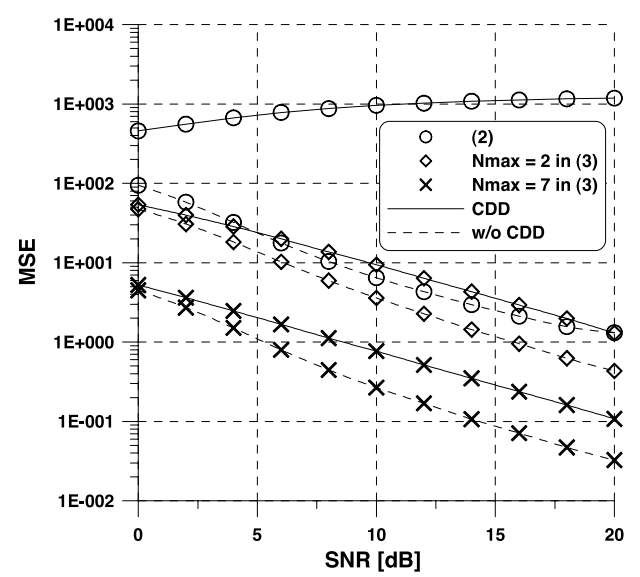

(a)

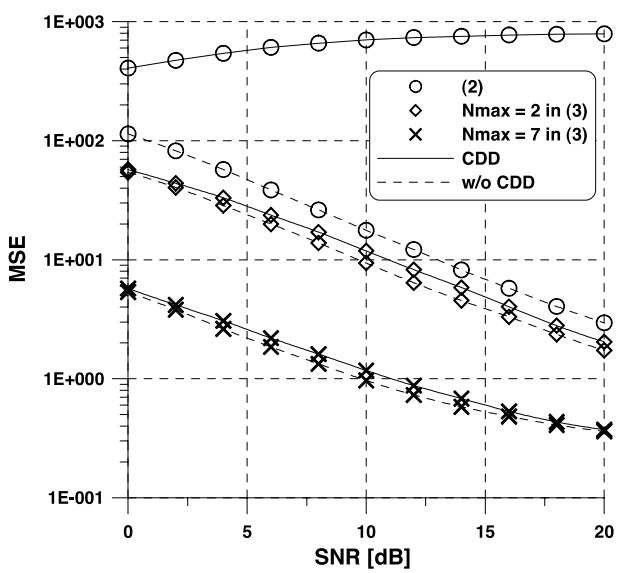

(b)

Fig. 1. MSE of residual STO estimation schemes with or without the use of CDD: (a) urban (b) terrain obstructed.

\section{Simulation results}

In this section, simulations are performed to verify the usefulness of the proposed estimation scheme under urban and terrain obstructed channels with maximum channel delays $3 \mu s$ and $16 \mu s$, respectively, which is widely used in analyses of FM performance [10], considering $N=213, N_{g}=24$, $N_{t r c}=21, \mathrm{BW}=96 \mathrm{KHz}$, and center frequency is $90 \mathrm{MHz}[1,2]$. Also, $N_{T}=2$ and $|\tau| \leq N_{g} / 10$ are used in our simulation. To benchmark the performance of the proposed scheme, the conventional residual STO estimator in $[8,9]$ is considered. For cyclic delay $\delta_{2}$ 's, $\delta_{2}=71$ is used.

Figure 1 shows the mean squared error (MSE) of the conventional and proposed estimation schemes under urban and terrain obstructed channels. As expected, the conventional estimation scheme (2) fails to correctly estimate STO when CDD is used because of the impact of the frequency selectivity of the channel, when compared to the case without using the CDD, while the proposed estimation scheme (3) effectively deals with the symbol timing error regardless of the use of CDD and is implemented with reduced complexity, confirmed by Table II, at the cost of estimation range. Note that the estimation ranges of (3) when $N_{\max }=2$ and 7 are limited by \pm 11.83 and \pm 3.944 in sample unit, respectively.

Figure 2 compares the variance and MSE of residual STO estimation schemes versus $N_{\max }$. In the urban channel, one can see that $E\{\hat{\tau}\}=\tau$ because the MSE of an unbiased estimator is just its variance. On the other hand, the estimation performance of (3) is deteriorated with the increase 


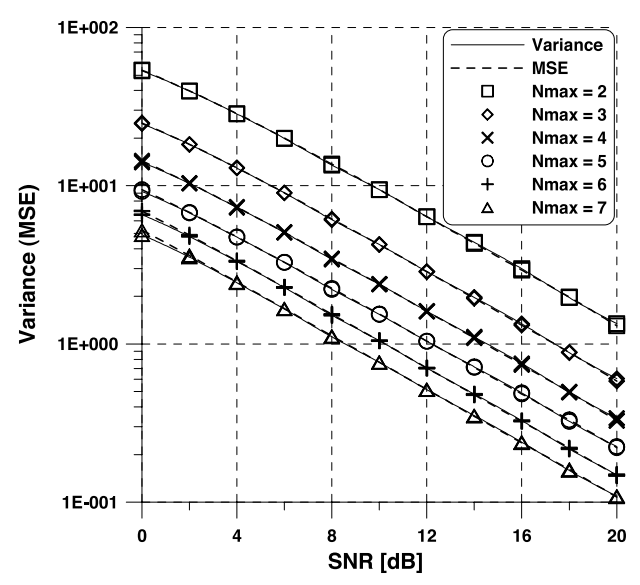

(a)

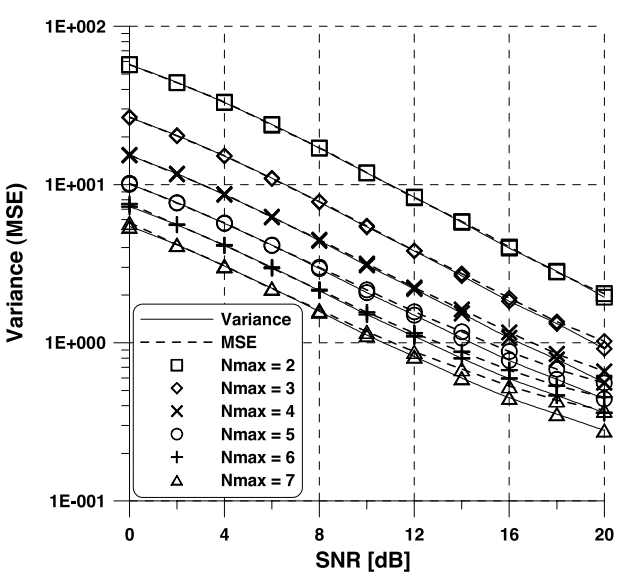

(b)

Fig. 2. Variance and MSE of the proposed residual STO estimation scheme versus $N_{\max }$ : (a) urban (b) terrain obstructed.

in maximum channel delays and $E\{\hat{\tau}\} \neq \tau$ at the high SNR, because the approximation $H\left(k_{i}\right) \approx H\left(k_{j}\right)$ is weakened, still being better than that of the conventional scheme.

\section{Conclusion}

This paper proposed a way into designing a simple and robust residual SFO estimation algorithm in the OFDM-based DRM+ system using CDD scheme. By utilizing the pilot partitioning and cyclic delay selection, the proposed STO estimator was made to keep low computational cost and be robust to the frequency selectivity of the channel. The simulation results of the STO estimation scheme in the DRM+ receiver with CDD established the validity of its effectiveness in the digital FM broadcasting application.

\section{Acknowledgments}

This research is supported by the ubiquitous Computing and Network (UCN) Project, the Ministry of Knowledge and Economy (MKE) Knowledge and Economy Frontier R\&D Program in Korea, and is supported by Seoul R\&BD Program. 